JOURNAL DE PHYSIQUE IV

Colloque C2, supplément au Journal de Physique III, Volume 5, février 1995

\title{
Micromechanical Modelling of the Superelastic Behavior
}

E. Patoor, A. Eberhardt, M. Berveiller

Laboratoire de Physique et Mécanique des Matériaux, URA 1215 du CNRS, Institut Supérieur de Génie Mécanique et Productique, Université de Metz, Ile du Saulcy, 57045 Metz cedex, France

\begin{abstract}
Shape Memory behavior in polycrystalline material is very sensitive to the local stress state. To take this features into account, we have developed a micromechanical approach based on a kinematical description of the physical strain mechanisms and a definition of a local thermodynamical potential. Volume fractions of the different variants of martensite are chosen as internal variables to describe the evolution of the microstructural state of the material. Physical limitations excerted on these variables are accounted using a constrained potential. Dissipative aspects at the origin of the hysteretic behavior of these alloys, need to define a dissipative potential. This analysis determines the local constitutive equations for the behavior. Global relationship are determined using a self consistent approach. Results obtained by this way are in good agreement with experimental observations performed on Cu-based Shape Memory alloys. In addition, this modelling is able to determine the kinetics of the phase transition and to give the evolution of the transformation strain for different thermomechanical loading paths.
\end{abstract}

\section{INTRODUCTION}

Phase transitions occuring in solids state produce large effects on the thermomechanical behavior. Shape memory alloys are an interesting case because it is reasonnable to assume that the martensitic transformation is the only inelastic strain mechanism. An accurate description of this strain mechanism is needed to account shape memory behavior. To illustrate this point, this paper deals with two different microstructure descriptions. A global one, using a two-phases approach leads to similar results than some phenomenological modelling [1,2] (lack of information on the transformation strain, oblique line). These troubles are avoid taking account the granular structure of the material and the existence of several variants inside the martensitic phase [3]. In this last approach, accurate constitutive equations are obtained in the single crystal case and applied to a polycrystalline material using a homogeneization method. Results obtained in that way well captured experimental features. Such approach gives information on the microstructure evolution during the loading process and can be successfully applied to complex loading conditions.

\section{KINEMATICAL AND THERMODYNAMICAL ASPECTS}

In Shape Memory Alloys, the total strain field is composed by thermal, plastic and elastic ( $\left.\varepsilon^{e}\right)$ componants and by a large inelastic reversible transformation strain $\varepsilon^{\mathrm{T}}$. In the infinitesimal deformation framework these contributions acts in an additive way. At first approximation, one may consider that no plasticity occurs during a thermoelastic transformation. This is related to the very few volume change associated to this kind of transformation. In order to focus our attention on the transformation strain, the elastic complience tensor $\mathrm{M}$ is assumed to be isotropic and uniform throught the material. In the same way, the thermal expansion factor and the temperature field are also regarded as uniform. 
According to these previous assumptions, total strain $E$ may be decomposed into several contributions. The overall transformation strain $\mathrm{E}^{\mathrm{T}}$ is then defined by :

$$
E_{i j}^{T}=\frac{1}{V} \int_{V} \varepsilon_{i j}^{T}(r) d V
$$

Evolution of this strain is obtained from the definition of a thermodynamical potential function of the control parameters (applied stress $\Sigma$ and temperature $T$ ) and a set of internal variables $\mathrm{Y}^{\mathrm{K}}$. Let us consider a reference volume $V$ (bounded by $\partial V$ ) of parent phase such as given surface forces applied on $\partial V$ are large enougth to form a volume $V_{M}$ of martensite. Complementary free energy $\Psi\left(\Sigma, T, Y^{K}\right)$ is then constituted by elastic energy $W_{\text {elas }}$, chemical energy $\Delta G_{\text {ch }}$ and by interfacial energy $W_{\text {int }}$.

$$
\Psi\left(\Sigma_{\mathrm{ij}}, \mathrm{T}, \mathrm{Y}^{\mathrm{K}}\right)=-\left[\Delta \mathrm{G}_{\mathrm{ch}}+\mathrm{W}_{\mathrm{elas}}+\mathrm{W}_{\mathrm{int}}-\Sigma_{\mathrm{ij}} \mathrm{E}_{\mathrm{ij}}\right]
$$

Chemical energy depends on the temperature ; a linear approximation around $T_{0}$ the thermodynamical equilibrium temperature is commonly used [4]. The elastic energy is determined using the classical relation.

$$
\Delta \mathrm{G}_{\mathrm{ch}}(\mathrm{T})=\mathrm{B}\left(\mathrm{T}-\mathrm{T}_{0}\right) \mathrm{V}_{\mathrm{M}} \quad \text { and } \quad \mathrm{W}_{\mathrm{elas}}=\frac{1}{2} \int_{\mathrm{v}} \sigma_{\mathrm{ij}}(\mathrm{r}) \varepsilon_{\mathrm{ij}}^{\mathrm{e}}(\mathrm{r}) \mathrm{dV}
$$

Due to incompatibilities in the transformation strain field, local stress $\sigma(\mathbf{r})$ must be divided into applied and internal stress $\sigma^{\mathrm{int}}$ (that is only related to the incompatibilities). Integration by parts and boundary conditions imposed on $\partial \mathrm{V}$ give the following expression for the elastic energy :

$$
\mathrm{W}_{\mathrm{elas}}=\frac{1}{2} \mathrm{~V} \Sigma_{\mathrm{ij}} \mathbf{M}_{\mathrm{ijkl}} \Sigma_{\mathrm{kl}}-\frac{1}{2} \int \sigma_{\mathrm{ij}}^{\mathrm{ink}}(\mathrm{r}) \varepsilon_{\mathrm{ij}}^{\mathrm{T}}(\mathrm{r}) \mathrm{dV}
$$

In the propagation stage of the transformation, the oblate shape of the martensite plates leads surface energy to be negligible in regard of the elastic one. According to the assumption of an isochoric transformation, the complementary free energy per unit reference volum is expressed by :

$$
\Psi\left(\Sigma_{\mathrm{ij}}, \mathrm{T}, \mathrm{Y}^{\mathrm{K}}\right)=\frac{1}{2} \Sigma_{\mathrm{ij}} \mathrm{M}_{\mathrm{ijk} \mathbf{l}} \Sigma_{\mathrm{kl}}+\Sigma_{\mathrm{ij}} \mathrm{E}_{\mathrm{ij}}^{\mathrm{T}}-\mathrm{B}\left(\mathrm{T}-\mathrm{T}_{0}\right) \frac{\mathrm{V}_{\mathrm{M}}}{\mathrm{V}}+\frac{1}{2 \mathrm{~V}} \int_{\mathrm{v}} \sigma_{\mathrm{ij}}^{\mathrm{int}}(\mathrm{r}) \varepsilon_{\mathrm{ij}}^{\mathrm{T}}(\mathrm{r}) \mathrm{dV}
$$

Different point of wiew may be adopted to described the microstructural aspects related to the phase transition in relations (1) and (5). Two of these approaches are detailled in this paper. The more global one consider the material as a mixture of two phases.

\section{TWO PHASES APPROXIMATION}

In that macroscopic approximation, where only averaging properties are taken into account, internal state of the material can be specified using only two internal variables [5]. One is the volumic fraction of product phase $\left(f=V_{M} / V\right)$, the other is the average transformation strain $\bar{\varepsilon}^{T}$ defined from :

$$
E_{i j}^{T}=\frac{V_{M}}{V} \frac{1}{V_{M}} \int_{V_{M}} \varepsilon_{i j}^{T}(r) d V=f \bar{\varepsilon}_{i j}^{T}
$$

Due to the physical meaning of these parameters, they are submitted to kinematical constraints. Obviously, $f$ is positive and smaller than unity. But there is no trivial way to express physical restrictions on the mean transformation strain, some additional assumptions are then necessary to account them.

Supplementary hypothesis are also need to determine the behavior law. Considering strain as piecewise uniform turns the thermodynamical potentiel into [5]:

$$
\Psi\left(\Sigma_{i j}, T, f, \bar{\varepsilon}_{i j}^{T}\right)=\frac{1}{2} \Sigma_{i j} M_{i j k l} \Sigma_{k l}+\Sigma_{i j} f \bar{\varepsilon}_{i j}^{T}-B\left(T-T_{0}\right) f+A_{i j k l} f(1-f) \bar{\varepsilon}_{k l} \bar{\varepsilon}_{k l}
$$

where $A$ is a constant tensorial quantity related to the elastic properties and to the morphology of the martensite phase. This particular form (7) defines an oblique line for the behavior (coming from the $f(1-f)$ contribution as reported by $[1,2]$ ). Such results have no physical sense because it is equivalent to assume that internal stress turns to zero in the martensitic state.

It is possible to avoid such troubles using a more accurate description for the microstructure dealing with the polycrystalline nature of the material and with the possibility to form several variants of the same martensitic phase. This is the object of the following description. 


\section{CRYSTALLOGRAPHICAL APPROACH FOR THE SINGLE CRYSTAL}

At this microstructural level, variants are characterized by a habit plane and a direction of transformation. Transformation strain $\varepsilon^{\mathrm{n}}$ associated to the formation of a variant $\mathrm{n}$ is linked to these characteristics and to the amplitude $\mathrm{g}$ of the transformation strain that is equal for all. Overall transformation strain $\varepsilon^{\mathrm{Tr}}$ for a crystal is obtained considering $\varepsilon^{\operatorname{Tr}}(r)$ as piecewise uniform. Introducing the volume fraction $\left(\mathrm{f}^{\mathrm{n}}=\mathrm{V}^{\mathrm{n}} / \mathrm{V}\right)$ of each variant gives:

$$
\varepsilon_{i j}^{T_{i j}}=\frac{1}{V} \int_{V} \sum_{n} \varepsilon_{i j}^{n} \theta^{n}(r) d V=\sum_{n} \varepsilon_{i j}^{n} f^{n} \quad \text { with } \quad \theta^{n}(r)=1 \text { if } r \in V^{n}\left(=0 \text { if } r \notin V^{n}\right)
$$

Physical limitations excerted on $\mathrm{f}^{\mathrm{n}}$ are obviously determined as in the previous section.

$$
\mathrm{f}^{\mathrm{n}} \geq 0 \quad \text { and } \quad \sum_{\mathrm{n}} \mathrm{f}^{\mathrm{n}}=\mathrm{f} \leq 1
$$

But this description do not need additionnal condition on the transformation strain. In the same way it is possible to obtain a more realistic evaluation for the interaction energy related with the internal stress field. Considering transformation strain field as piecewise uniform turns this contribution into :

$$
E_{i n t}=-\frac{1}{2 V} \int_{v} \sigma_{i j}^{i n t}(r) \varepsilon_{i j}^{T r}(r) d V=-\frac{1}{2 V} \int_{V} \sigma_{i j}^{i n t}(r) \sum_{n} \varepsilon_{i j}^{n} \theta^{n}(r) d V=-\frac{1}{2 V} \sum_{n} \varepsilon_{i j}^{n} \int_{v^{n}} \sigma_{i j}^{i n t}(r) d V
$$

where the integral of $\sigma^{\text {int }}$ over $\mathrm{V}^{\mathrm{n}}$ defines the mean internal stress inside the variant $\mathrm{n}$ which can be determined using the Eshelby-Kröner approach [6]. Denoted by $\mathbf{C}$ the four-order elastic modulus, by $\mathbf{S}$ the Eshelby tensor and by I the four-order identity tensor, gives the following expression

$$
\int_{\mathrm{v}^{\mathrm{n}}} \sigma_{\mathrm{ij}}^{\mathrm{int}}(\mathbf{r}) \mathrm{dV}=\mathrm{C}_{\mathrm{jkl}}\left(\mathrm{d}_{\mathrm{klpq}}-\mathrm{S}_{\mathrm{klpq}}^{\mathrm{n}}\right)\left(\sum_{\mathrm{m}} \varepsilon_{\mathrm{pq}}^{\mathrm{m}} \mathrm{f}^{\mathrm{m}}-\varepsilon_{\mathrm{pq}}^{\mathrm{n}}\right)
$$

Equation (11) leads to consider that the volumic fractions $\mathrm{f}^{\mathrm{m}}$ and the geometry characteristics $\mathrm{S}^{\mathrm{n}}$ for the martensitic plates (shape and orientation) are the relevant parameters to control the interaction energy associated to a martensitic microstructure. Neverveless, to take this complete set of internal parameters into the complementary free energy is to much complicated. Previous micromechanical analysis $[7,8]$ have shown that this resistive action can be represented using an interaction matrix $\mathrm{H}^{\mathrm{nm}}$ that includes the shape and orientation factors.

$$
E_{\text {int }}=\frac{1}{2} \sum_{n, m} H^{n m} f^{n} \mathbf{f}^{m}
$$

In fine variables $\mathrm{fm}^{\mathrm{m}}$ are then sufficient to describe evolution of the microstructural state and they are used as internal variables in the thermodynamical potential now expressed by :

$$
\Psi\left(\Sigma_{\mathrm{ij}}, \mathrm{T}, \mathrm{f}^{\mathrm{n}}\right)=\frac{1}{2} \Sigma_{\mathrm{ij}} \mathrm{M}_{\mathrm{ijkl}} \Sigma_{\mathrm{kl}}+\Sigma_{\mathrm{ij}} \sum_{\mathrm{n}} \varepsilon_{\mathrm{ij}}^{\mathrm{n}} \mathrm{f}^{\mathrm{n}}-\mathrm{B}\left(\mathrm{T}-\mathrm{T}_{0}\right) \mathrm{f}-\frac{1}{2} \sum_{\mathrm{n}, \mathrm{m}} \mathrm{H}^{\mathrm{nm}} \mathrm{f}^{\mathrm{n}} \mathrm{f}^{\mathrm{m}}
$$

Driving force $\mathrm{F}^{\mathrm{n}}$ acting on internal variable $\mathrm{f}^{\mathrm{n}}$ is obtained from the partial derivative of $\Psi$ with respect to these internal variables [9]. Existence of kinematical constraints (9) imposes to introduce a Lagrangian functional $L\left(\Sigma_{i j}, T, f^{i}\right)[10]$ and to use the Kuhn-Tucker optimaly conditions.

$$
L\left(\Sigma_{\mathrm{ij}}, \mathrm{T}, \mathrm{f}^{\mathrm{n}}\right)=\Psi\left(\Sigma_{\mathrm{ij}}, \mathrm{T}, \mathrm{f}^{\mathrm{n}}\right)-\lambda_{0}\left[\sum_{\mathbf{n}} \mathrm{f}^{\mathrm{n}}-1\right]-\sum_{\mathrm{n}} \lambda_{\mathrm{n}}\left[-\mathrm{f}^{\mathrm{n}}-0\right]
$$

where Lagrange multiplier $\lambda_{0}$ (resp. $\lambda_{\mathrm{n}}$ ) is a positive quantity associated with the inequality constraints $\mathrm{f} \leq$ 1 (resp. $\mathrm{f}^{\mathrm{n}} \leq 0$ ). Definition of the driving force turns into:

$$
\mathrm{F}^{\mathrm{n}}=\Sigma_{\mathrm{ij}} \varepsilon_{\mathrm{ij}}^{\mathrm{n}}-\mathrm{B}\left(\mathrm{T}-\mathrm{T}_{0}\right)-\sum_{\mathrm{m}} \mathrm{H}^{\mathrm{nm}} \mathrm{f}^{\mathrm{m}}-\lambda_{0}+\lambda_{\mathrm{n}}
$$

Despite the thermoelastic character of the martensitic transformation in Shape Memory Alloys, the thermomechanical behavior of these material exhibit an hysteretic effect. Thus their behavior can not be specified using the thermodynamical potential alone. The definition of a pseudo-dissipative potential $\mathrm{W}^{\mathrm{d}}$ is required. 


\section{PSEUDO-DISSIPATIVE POTENTIAL AND CONSTITUTIVE EQUATIONS}

The superelastic behavior of Shape Memory Alloys is then determined considering that the driving force $\mathrm{F}^{\mathrm{n}}$ must reach a critical value to produce growing or shrinkage of a variant. This critical value $F_{c}$ is related to the microstructural state of the material. It is reasonnable to assume the resistive dissipative force equals on all the variant. Conditions necessaries to obtain a transformation flow are then established.

$$
\begin{array}{llll}
\dot{\mathrm{f}}^{\mathrm{n}}=0 & \text { if } & \mathrm{F}^{\mathrm{n}}<\mathrm{F}_{\mathrm{c}} & \forall \\
\dot{\mathrm{f}}^{\mathrm{n}}=0 & \text { if } & \mathrm{F}^{\mathrm{n}}=\mathrm{F}_{\mathrm{c}} & \text { and } \dot{\mathrm{F}}_{\mathrm{c}}=0 \\
\dot{\mathrm{f}}^{\mathrm{n}} \neq 0 & \text { if } & \mathrm{F}^{\mathrm{n}}=\mathrm{F}_{\mathrm{c}} & \text { and } \dot{\mathrm{F}}_{\mathrm{c}}=\dot{\mathrm{F}}^{\mathrm{n}}
\end{array}
$$

$F_{c}$ is a positive material constant. Second law of the thermodynamics and the energy balance require:

$$
\dot{\Psi}_{\mid \Sigma, T}=\dot{W}^{d}=\sum_{n} F^{n} \dot{f}^{n} \geq 0
$$

If there are no coupling effect for dissipation on each variant, different expressions for forward and reverse transformation are thus obtained.

$$
A->M \Rightarrow F^{n} \dot{f}^{n}=F_{c} \dot{f}^{n} \text { and } M->A \Rightarrow F^{n} \dot{f}^{n}=-F_{c} \dot{f}^{n}
$$

From expression (15) and conditions (20), each variant of martensite has to observe local transformation criteria, defined in the forward and reverse case.

$$
\Sigma_{\mathrm{ij}} \varepsilon_{\mathrm{ij}}^{\mathrm{n}}-\mathrm{B}\left(\mathrm{T}-\mathrm{T}_{0}\right)-\sum_{\mathrm{m}} \mathrm{H}^{\mathrm{nm}} \mathbf{f}^{\mathrm{m}}-\lambda_{0}+\lambda_{\mathrm{n}}={ }_{-}^{+} \mathrm{F}_{\mathrm{c}}
$$

Kinematical conditions (9) impose to verify $\mathrm{n}+1$ additional relations:

$$
\begin{aligned}
& \lambda_{0}=\Sigma_{\mathrm{ij}} \varepsilon_{\mathrm{jj}}^{\mathrm{n}}-\mathrm{B}\left(\mathrm{T}-\mathrm{T}_{0}\right)-\sum_{\mathrm{m}} \mathrm{H}^{\mathrm{nm}} \mathrm{f}^{\mathrm{m}}+\lambda_{\mathrm{n}}{ }_{-}^{+} \mathrm{F}_{\mathrm{c}} \geq 0 \\
& \lambda_{\mathrm{n}}=-\Sigma_{\mathrm{ij}} \varepsilon_{\mathrm{ij}}^{\mathrm{n}}+\mathrm{B}\left(\mathrm{T}-\mathrm{T}_{0}\right)+\sum_{\mathrm{m}} \mathrm{H}^{\mathrm{nm}} \mathrm{f}^{\mathrm{m}}+\lambda_{0} \stackrel{+}{{ }^{+} \mathrm{F}_{\mathrm{c}} \geq 0}
\end{aligned}
$$

If criteria (21) and conditions (22) are satisfied, the evolution of $\mathrm{f}^{\mathrm{n}}$ is determined according to the consistency condition. Resolution of this system equations determines the following constitutive equation for the transformation strain rate applied to a unit volume of crystal.

$$
\dot{\varepsilon}_{\mathrm{ij}}^{\mathrm{Tr}}=\sum_{\mathbf{n}} \varepsilon_{\mathrm{ij}}^{\mathbf{n}} \sum_{\mathbf{m}}\left(\mathrm{H}^{\mathrm{nm}}\right)^{-1}\left[\varepsilon_{\mathrm{kl}}^{\mathbf{m}} \dot{\Sigma}_{\mathbf{k} 1}-\mathrm{B} \dot{\mathrm{T}}\right]
$$

Set of equations $(21,22,23)$ characterizes the transformation plasticity in the single crystal case. In a freestress state, using criteria (21) allows to define the Martensite start temperature $\mathbf{M}_{\mathrm{s}}$ and the Austenite finish temperature $\mathbf{A}_{\mathrm{f}}$.

$$
M_{s}=T_{0}-\frac{F_{c}}{B} \quad \text { and } \quad A_{f}=T_{0}+\frac{F_{c}}{B}
$$

These two temperatures are only function of some intrinsic parameters of the transformation, they can be used to characterize the material behavior and to give an experimental value for $F_{c}$.

\section{POLYCRYSTALLINE BEHAVIOR}

Previous relations are determined in a single crystal condition, where crystallographical orientations are well known and where loading condition can be assumed to be uniform inside the specimen. Such accurate analysis is impossible in the polycrystalline case. Local loading condition is now unknown due to the existence of an internal stress field associated to the granular structure. This additional difficulty can be solved using homogenization methods. Three fundamental equations are then employed at the micro level. First is the local equilibrium condition, second is the continuity of deformation and the last one is the local behavior (23) established in the previous section. 
A self-consistent approximation is chosen to compute the effective behavior [11]. This framework considers the effective overall medium as the homogeneous reference one. This medium is characterized by a set of uniform tangent modulus ( $\mathrm{L}^{\mathrm{eff}}$ and $\mathrm{M}^{\mathrm{eff}}$ ) and it is subject to an uniform strain $\mathrm{E}^{\mathrm{o}}$. From this definition local modulus are expressed using their deviation part to these uniform quantity.

$$
1_{i j k l}(r)=L_{i j k l}^{\text {eff }}+\delta 1_{i j k l}(r) \text { and } m_{i j}(r)=M_{i j}^{\text {eff }}+\delta m_{i j}(r)
$$

Introducing local behavior into equilibrium condition, and considering the uniformity of the properties inside the homogeneous reference medium and the assumption of uniformity for the temperature lead to :

$$
\mathrm{L}_{\mathrm{ijkl}}^{\text {eff }}\left(\dot{\varepsilon}_{\mathrm{kl}}(\mathrm{r})\right)_{\mathrm{j}}+\left(\delta \mathrm{l}_{\mathrm{j} \mathrm{jkl}}(\mathrm{r}) \dot{\varepsilon}_{\mathrm{kl}}(\mathrm{r})-\delta \mathrm{m}_{\mathrm{ij}}(\mathrm{r}) \dot{\mathrm{T}}\right)_{\mathrm{j}}=0
$$

Resolution of expression (25) using the Green tensor method gives the following integral equation :

$$
\dot{\varepsilon}_{\mathrm{mn}}(\mathrm{r})=\mathrm{E}_{\mathrm{mn}}^{\mathrm{o}}+\int_{\mathrm{v}} \Gamma_{\mathrm{mnij}}^{\mathrm{o}}\left(\mathrm{r}-\mathrm{r}^{\prime}\right)\left[\delta \mathrm{l}_{\mathrm{ijkl}}\left(\mathrm{r}^{\prime}\right) \dot{\varepsilon}_{\mathrm{kl}}\left(\mathrm{r}^{\prime}\right)-\delta \mathrm{m}_{\mathrm{ij}}\left(\mathrm{r}^{\prime}\right) \dot{\mathrm{T}}\right] \mathrm{dV^{ \prime }}
$$

where $\Gamma^{\circ}$ denotes the modified Green tensor. Resolution of this equation gives localisation expressions between local and overall strain. This determines the effective behavior of the polycristal.The overall constitutive relation is now completely defined from the knowledge of the internal structure evolution of the polycrystal.

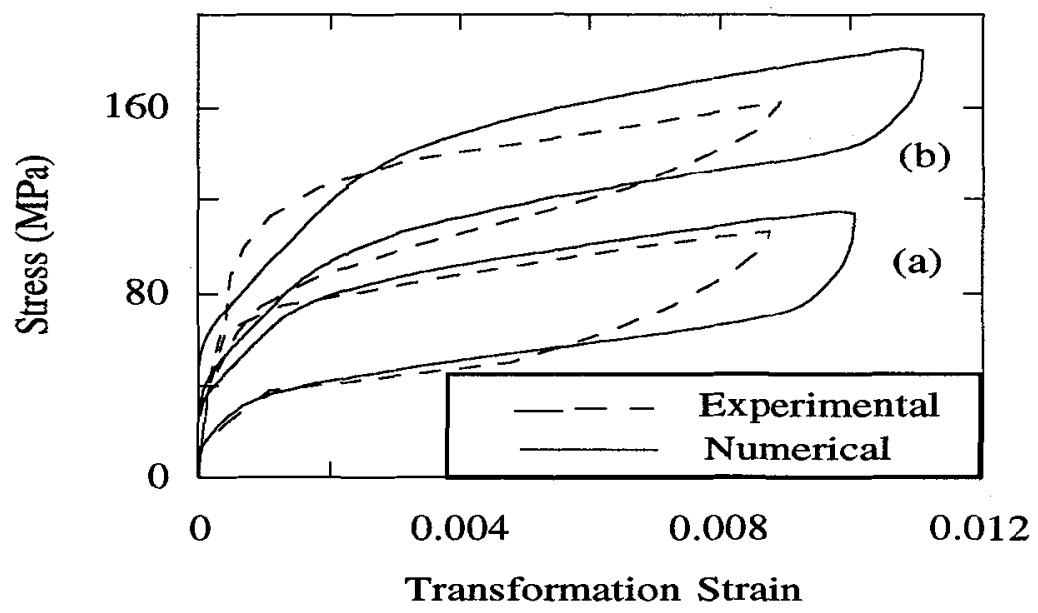

Figure 1: Influence of the temperature on the superelastic behavior. Comparison between experiment results [14] and self-consistent determination $\left(M_{s}=-97^{\circ} \mathrm{C}, A_{f}=-91^{\circ} \mathrm{C}, T_{a}=-80^{\circ} \mathrm{C}, T_{b}=-70^{\circ} \mathrm{C}\right)$.

\section{RESULTS}

Numerical results are obtained in that way. They are applied to $\mathrm{Cu}-\mathrm{Zn}-\mathrm{Al}$ Shape memory alloy. In these alloys 24 variants of martensite forming six self-accommodated groups, are observed. Direction for habit plane normal and transformation direction are 2-11-12 type. Transformation displacement $\mathrm{g}$ is about 0.23 [12]. Experimental determination performed with tensile test experiment on single crystal specimen gives $B \approx$ $0.23 \mathrm{MPa} \cdot \mathrm{K}^{-1}$ [13]. Hysteretic effect characterized by $F_{c}$ is determined from $M_{s}$ and $A_{f}$ temperatures. Interaction matrix $H^{m n}$ is constituted with two types of terms: weak one $\left(H^{1}=\mu / 1000\right)$ for self accommodated variants and strong one $\left(\mathrm{H}^{2}=\mu / 150\right.$ ) for the other (with $\mu \approx 40 \mathrm{GPa}$ ). The polycrystalline structure is described using 100 different grain orientations randomly chosen to induce no particular texture effect. Grain shape is assumed to be spherical. Result obtained in that way are successfully compared with experimental superelastic tensile test performed by P. Vacher [14] at two different temperatures (figure 1). 
From the same experimental data computed and measured transformation kinetics are compared (figure 2). Experimental features are well-captured by the self-consistent model presented here.

In addition this micromechanical scheme is able to give evolution of some macroscopic variables with the loading parameters. This is illustrated here for the mean transformation strain (figure 3) This quantity decreases from a maximal value associated to the formation of well-oriented variants, in the begining of the stress-induced transformation, to a constant value around $4 \%$, that is experimentaly observed in $\mathrm{Cu}-\mathrm{Zn}-\mathrm{Al}$ Shape Memory Alloys.

\section{CONCLUSION}

Numerical results obtained from the micromechanical approach presented in this paper are in very good agreement with experimental observations. Such results are obtained without any fitting parameter. The input data used in this framework are experimentally determined from measurement on single crystal and from texture analysis. These determinations are independent with the loading conditions. In addition, the proposed model well represented the dissymmetry experimentally observed between tensile and compressive test. Extension of such result for other loading conditions is able to determine a transformation criterion for macroscopic modelling. This point is on first importance for engineering applications [15].

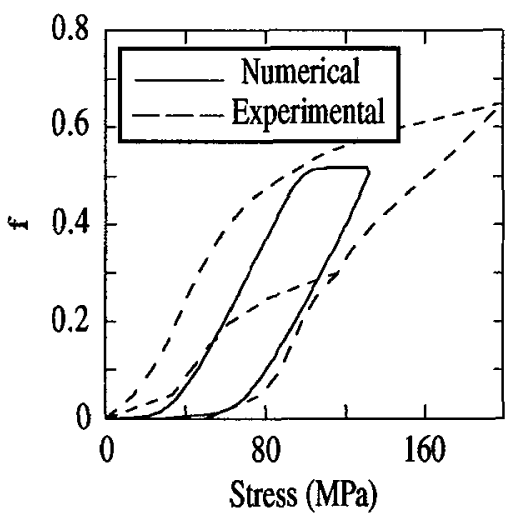

Figure 2: Kinetics of a stress-induced transformation. Comparison between experiment results [14] and self-consistent determination for tensile test at $\mathrm{T}=-80^{\circ} \mathrm{C}\left(\mathrm{M}_{\mathrm{s}}=-97^{\circ} \mathrm{C} \mathrm{A}_{\mathrm{f}}=-91^{\circ} \mathrm{C}\right)$.

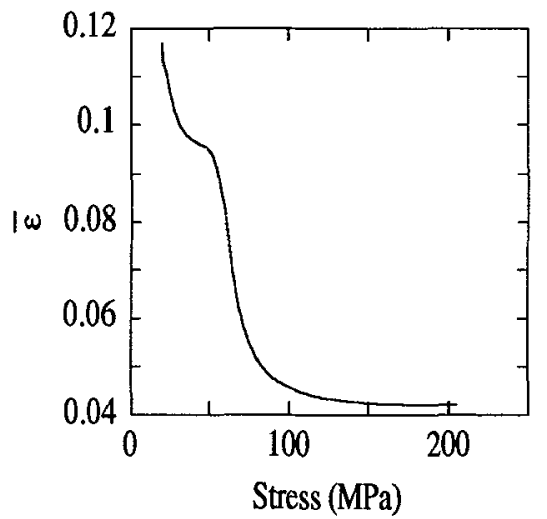

Figure 3: Evolution of the mean Transformation Strain for an uniaxial tensile test experiment.

\section{References.}

[1] Raniecki B., Lexcellent C. and Tanaka K., Arch.Mech., 3, (1992) 261-284 .

[2] Sun Q.P. and Hwang K. C., J. Mech. Phys. Solids, 41 (1993) 1.

[3] Patoor E., Eberhardt A., Berveiller M., Pitman Research Notes in Math. Series, 296 (1993) 38-54.

[4] Ortin J. and Planes A., Acta Metall., 36 (1988) 1873.

[5] Patoor E., Bensalah M.O., Eberhardt A., Berveiller M., ICOMAT"92, Monterey, CA., USA, 401-

[6] Mura T., Micromechanics of defects in solids, (Martinus Nijhoff, The Hague 1987).

[7] Fassi Fehri O., Hihi A., Berveiller M., Scripta Met., 21 (1987) 771.

[8] Bensalah M.O., Thèse d'État, Université Mohammed V, Rabat (1992), Maroc.

[9] Rice J.R., J.Mech. Phys. Solids, 19 (1971) 433.

[10] Quoc Son N., Procs. IJSS (1991).

[11] Lipinski P. and Berveiller M., Int.J. of Plasticity, 5 (1989) 149.

[12] De Vos J., Aernoudt E. and Delaey L., Z. Metallkde, 69 (1978) 438-444.

[13] Patoor E., Eberhardt A. and Berveiller M., Arch. Mech., 40 (1988) 775-794.

[14] Vacher P., Thèse, Université de Franche-Comté, Besançon (1991) France.

[15] Patoor E., El Amrani M., Eberhardt A. and Berveiller M., this conf. 